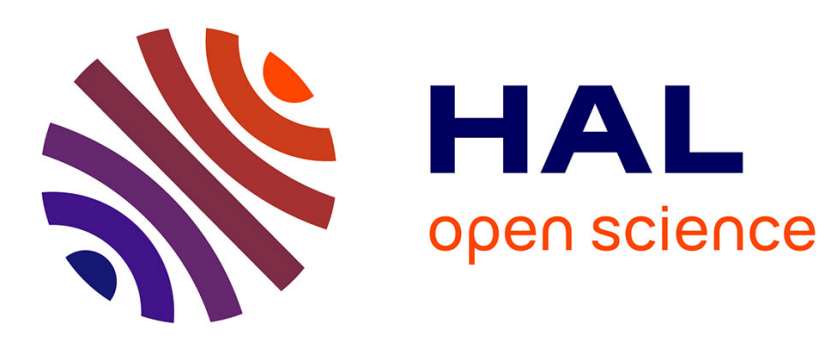

\title{
Pathwidth of outerplanar graphs
}

David Coudert, Florian Huc, Jean-Sébastien Sereni

\section{To cite this version:}

David Coudert, Florian Huc, Jean-Sébastien Sereni. Pathwidth of outerplanar graphs. Journal of Graph Theory, 2007, 55 (1), pp.27 - 41. 10.1002/jgt.20218 . inria-00429216

\section{HAL Id: inria-00429216 https://hal.inria.fr/inria-00429216}

Submitted on 1 Nov 2009

HAL is a multi-disciplinary open access archive for the deposit and dissemination of scientific research documents, whether they are published or not. The documents may come from teaching and research institutions in France or abroad, or from public or private research centers.
L'archive ouverte pluridisciplinaire HAL, est destinée au dépôt et à la diffusion de documents scientifiques de niveau recherche, publiés ou non, émanant des établissements d'enseignement et de recherche français ou étrangers, des laboratoires publics ou privés. 


\title{
Pathwidth of outerplanar graphs *
}

\author{
David Coudert, Florian Huc and Jean-Sébastien Sereni \\ MASCOTTE, I3S-CNRS-INRIA-UNSA \\ 2004 route des Lucioles - BP93 \\ 06902 Sophia Antipolis, FRANCE \\ E-mail: lastnamedsophia.inria.fr
}

\begin{abstract}
We are interested in the relation between the pathwidth of a biconnected outerplanar graph and the pathwidth of its (geometric) dual. Bodlaender and Fomin [3], after having proved that the pathwidth of every biconnected outerplanar graph is always at most twice the pathwidth of its (geometric) dual plus two, conjectured that there exists a constant $c$ such that the pathwidth of every biconnected outerplanar graph is at most $c$ plus the pathwidth of its dual. They also conjectured that this was actually true with $c$ being one for every biconnected planar graph. Fomin [10] proved that the second conjecture is true for all planar triangulations. First, we construct for each $p \geq 1$ a biconnected outerplanar graph of pathwidth $2 p+1$ whose (geometric) dual has pathwidth $p+1$, thereby disproving both conjectures. Next, we also disprove two other conjectures (one of Bodlaender and Fomin [3], implied by one of Fomin [10]). Finally we prove, in an algorithmic way, that the pathwidth of every biconnected outerplanar graph is at most twice the pathwidth of its (geometric) dual minus one. A tight interval for the studied relation is therefore obtained, and we show that all cases in the interval happen.
\end{abstract}

\section{Introduction}

A planar graph is a graph that can be embedded in the plane without crossing edges. It is said to be outerplanar if it can be embedded in the plane without crossing edges and such that all its vertices are incident to the unbounded face. For any graph $G$, we denote by $V(G)$ its vertex set and by $E(G)$ its edge set. The dual of the planar graph $G$, denoted by $G^{*}$, is the graph obtained by putting one vertex for each face, and joining two vertices if and only if the corresponding faces are adjacent. The weak dual of $G$, denoted by $\mathcal{T}_{G}$, is the induced subgraph of $G^{*}$ obtained by removing the vertex corresponding to the unbounded face. As is well known, the weak dual of an outerplanar graph is a forest, and the weak dual of a biconnected outerplanar graph is a tree. Furthermore, linear-time algorithms to recognise and embed outerplanar graphs are known (see for instance [15, 21]). Note that the dual of a planar graph can also be computed in linear-time.

The notion of pathwidth was introduced by Robertson and Seymour [17]. A path decomposition of a graph $G=(V, E)$ is a set system $\left(X_{1}, \ldots, X_{r}\right)$ of $V$ such that

(i) $\bigcup_{i=1}^{r} X_{i}=V$;

(ii) $\forall x y \in E, \exists i \in\{1,2, \ldots, r\}:\{x, y\} \subset X_{i}$;

*This work was partially funded by the European projects IST FET AEOLUS and COST 293 GRAAL, and done within the CRC CORSO with France Telecom R\&D. 
(iii) $\forall\left(i_{0}, i_{1}, i_{2}\right) \in\{1,2, \ldots, r\}^{3}, i_{0}<i_{1}<i_{2} \Rightarrow X_{i_{0}} \cap X_{i_{2}} \subseteq X_{i_{1}}$.

The width of the path decomposition $\left(X_{1}, \ldots, X_{r}\right)$ is $\max _{1 \leq i \leq r}\left|X_{i}\right|-1$. The pathwidth of $G$, denoted by $\operatorname{pw}(G)$, is the minimum width over its path decompositions.

The pathwidth of a graph was shown to be equal to its vertex separation [12]: a layout (or vertexordering) $L$ of a graph $G=(V, E)$ is a one-to-one correspondence between $V$ and $\{1, \ldots,|V|\}$. The vertex separation of $(G, L)$ is $\max _{1 \leq i \leq|V|}|M(i)|$ where

$$
M(i):=\{v \in V: L(v)>i \text { and } \exists u \in N(v): L(u) \leq i\} .
$$

The vertex separation of $G$, denoted by vs $(G)$, is the minimum of the vertex separation of $(G, L)$ taken over all vertex-orderings $L$.

Computing the pathwidth of graphs is an active research area, in which a lot of work has been done (survey papers are for instance [6, 2, 16]). It was shown [4] that the pathwidth of graphs with bounded treewidth can be computed in polynomial time. As outerplanar graphs have treewidth two, the pathwidth of an outerplanar graph is polynomially computable. However, the exponent in the running time of the algorithm is rather large, so the algorithm is not useful in practice. This is why Govindan et al. [11] gave an $O(n \log (n))$ time algorithm for approximating the pathwidth of outerplanar graphs with a multiplicative factor of three. For biconnected outerplanar graphs, Bodlaender and Fomin [3] improved upon this result by giving a linear-time algorithm which approximates the pathwidth of biconnected outerplanar graphs with a multiplicative factor two (and a corresponding path decomposition is obtained in time $O(n \log (n)))$. To do so, they exhibited a relationship between the pathwidth of an outerplanar graph and the pathwidth of its dual. More precisely, the following holds.

Theorem 1 (Bodlaender and Fomin [3]) Let $G$ be a biconnected outerplanar graph without loops and multiple edges. Then $\mathrm{pw}\left(G^{*}\right) \leq \mathrm{pw}(G) \leq 2 \mathrm{pw}\left(G^{*}\right)+2$.

Observe that adding a vertex linked to all other vertices of any graph increases its pathwidth by exactly one. Since the weak dual of an outerplanar graph (which can be computed in linear-time) is a tree and there exist linear-time algorithms to compute the pathwidth of a tree [8], this yields the desired approximation (obtaining a corresponding path decomposition needs more work).

Bodlaender and Fomin [3] suggested that a stronger relationship holds between the pathwidth of a planar graph and the pathwidth of its dual.

Conjecture 1 (Bodlaender and Fomin [3]) There is a constant c such that for every biconnected outerplanar graph $G$ without loops and multiple edges $\mathrm{pw}(G) \leq \mathrm{pw}\left(G^{*}\right)+c$.

Conjecture 2 (Bodlaender and Fomin [3]) For every biconnected planar graph $G$ without loops and multiple edges, $\mathrm{pw}(G) \leq \mathrm{pw}\left(G^{*}\right)+1$.

Fomin [10] proved that if $G$ is any biconnected planar graph of maximum degree at most three, then $\operatorname{pw}(G) \geq \mathrm{pw}\left(G^{*}\right)-1$. This implies that Conjecture 2 is true for every planar triangulation, since any planar triangulation is the dual of a biconnected planar graph of maximum degree three.

It is worth noting that these conjectures are motivated by the following result about the treewidth, conjectured by Robertson and Seymour [18] and proved by Lapoire [13] using algebraic methods (notice that Bouchitté, Mazoit and Todinca [5] gave a shorter and combinatorial proof of this result).

Theorem 2 (Lapoire [13]) For every planar graph $G, \operatorname{tw}(G) \leq \operatorname{tw}\left(G^{*}\right)$. 
In Section 2, we exhibit a family $\left(G_{p}\right)_{p \geq 1}$ of biconnected outerplanar graphs with maximum degree four such that $\operatorname{pw}\left(G_{p}\right)=2 p+1$ and $\operatorname{pw}\left(G_{p}^{*}\right)=p+1$, thereby disproving both conjectures. To construct these graphs, we introduce a general construction which actually allows us to prove the following result.

Theorem 3 For every integer $p \geq 1$ and every integer $k \in\{1,2, \ldots, p+1\}$, there exists a biconnected outerplanar graph of pathwidth $p+k$ whose weak dual has pathwidth $p$.

Let us mention here that Fomin and Thilikos [9] also disproved, independently, Conjecture 1.

Next, we disprove the following conjecture of Bodlaender and Fomin [3].

Conjecture 3 (Bodlaender and Fomin [3]) For every simple 2-connected planar graph $G, \mathrm{pw}(G) \geq$ $\operatorname{pw}\left(G^{*}\right)-1$.

In Section 3, we prove the following result which improves the upper bound given by Theorem 1.

Theorem 4 Let $G$ be a biconnected outerplanar graph without loops and multiple edges. Then $\operatorname{pw}(G) \leq 2 \mathrm{pw}\left(G^{*}\right)-1$.

As a consequence, the previous approximation for the pathwidth of biconnected outerplanar graphs is also improved. We give an algorithmic proof which allows to obtain a layout of the outerplanar graph $G$ considered (and whose vertex separation is hence at most $2 \mathrm{pw}(G)-1$ ).

Furthermore, Theorem 3 shows that this bound is best possible in general.

\section{Counter-examples}

In this section, we establish Theorem 3 and deduce the following corollary which disproves Conjectures 1 and 2 .

Corollary 1 For every integer $p \geq 1$, there exists a triangle-free biconnected outerplanar graph $G_{p}$ of maximum degree four whose pathwidth is $2 p+1$ such that the pathwidth of its dual is $p+1$.

For each $i \in\{1,2,3,4\}$, let $H_{i}$ be a biconnected outerplanar graph of pathwidth $p$ whose weak dual has pathwidth $p^{\prime}$. We shall describe a construction which yields a biconnected outerplanar graph $C\left(H_{1}, H_{2}, H_{3}, H_{4}\right)$ of pathwidth $p+2$ whose weak dual has pathwidth $p^{\prime}+1$. This construction will be illustrated by examples yielding the graphs $G_{p}$ of Corollary 1 .

A 4-cycle is called a square. Two squares are adjacent if they share exactly one edge. The degree of the square $S$ is the number of squares adjacent to $S$. Let the cross $K$ be the biconnected outerplanar graph consisting of four squares of degree one and one square of degree four (see Figure 1(a)).

For each $i \in\{1,2,3,4\}$, let $x_{i} y_{i}$ be an edge of $H_{i}$ incident to the unbounded face in an outerplanar embedding of $H_{i}$. This edge is chosen such that there exists an optimal layout $L$ of $\mathcal{T}_{H_{i}}$ where the vertex $v$ corresponding to the the bounded face incident to $x_{i} y_{i}$ fulfils $L(v)=\left|V\left(\mathcal{T}_{H_{i}}\right)\right|$ for $i \in\{2,3\}$. Notice that this is always possible, and directly follows from Theorem 6 cited in Section 3. For $i \in\{1,2\}$, we denote by $L_{i}$ an optimal layout of $H_{i}$, i.e. a layout with vertex separation $p$, and without loss of generality we assume that $L_{i}\left(x_{i}\right)<L_{i}\left(y_{i}\right)$.

Consider the cross $K$ of Figure 1(a). For each $i \in\{1,2,3,4\}$, the edge $e_{i}$ of $K$ is identified with the edge $x_{i} y_{i}$. We assume moreover that the vertices $x_{1}$ and $x_{2}$ are identified with the vertices $u_{1}$ and $u_{2}$ respectively (see Figure $1(\mathrm{~b})$ ). Notice that there is generally not a unique way to achieve this 


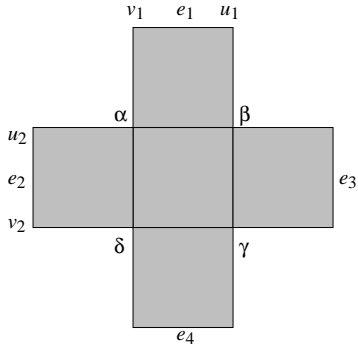

(a) The cross $K$.

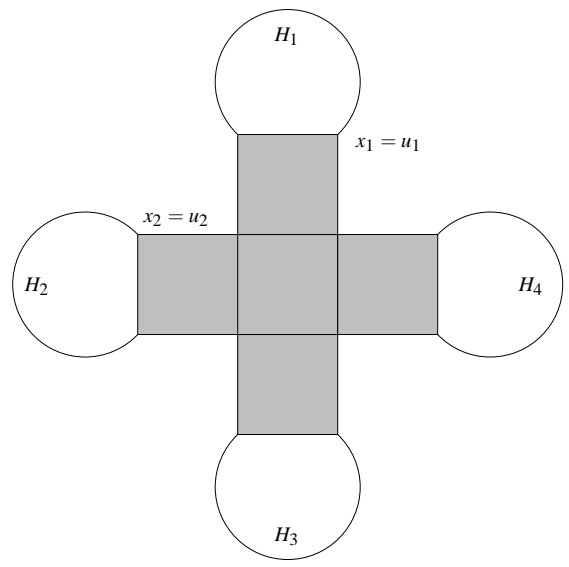

(b) Gluing four graphs $H_{1}, H_{2}, H_{3}, H_{4}$ on the cross $K$

Figure 1: When identifying the edges, we ensure that $x_{1}$ is identified with $u_{1}$ and $x_{2}$ with $u_{2}$.

construction, but we shall denote by $C\left(H_{1}, H_{2}, H_{3}, H_{4}\right)$ any graph obtained from $H_{1}, H_{2}, H_{3}, H_{4}$ in this way.

It is clear by the construction that any such graph $C\left(H_{1}, H_{2}, H_{3}, H_{4}\right)$ is a biconnected outerplanar graph. As an example, let $G_{1}$ be the biconnected outerplanar graph consisting of three squares of degree one and one square of degree three (see Figure 2). For any integer $p \geq 2$, let $G_{p}$ be the graph $\mathcal{C}\left(G_{p-1}, G_{p-1}, G_{p-1}, G_{p-1}\right)$, obtained as indicated in Figures 3 and 4 . Remark that the condition on the vertices $x_{1}$ and $x_{2}$ is clearly fulfilled in this case thanks to the symmetry of the graphs $G_{p}$, and that the maximum degree of $G_{p}$ is four.

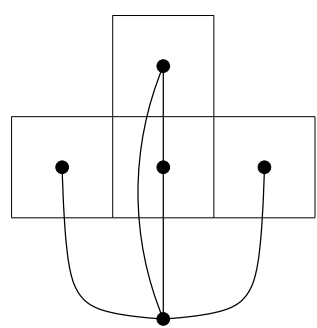

(a) $G_{1}$ and $G_{1}^{*}$

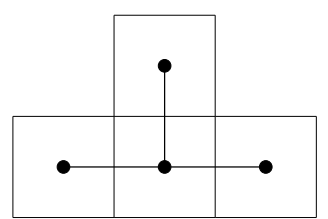

(b) $G_{1}$ and $\mathcal{T}_{G_{1}}$

Figure 2: $G_{1}$, graph consisting of one square of degree three and three squares of degree one, the dual $G_{1}^{*}$ and the weak dual $\mathcal{T}_{G_{1}}$, a star.

In the following three lemmata, we prove the announced properties of the construction. The central square of the cross is denoted by $S$, and the corresponding vertex of the dual is $s$. 


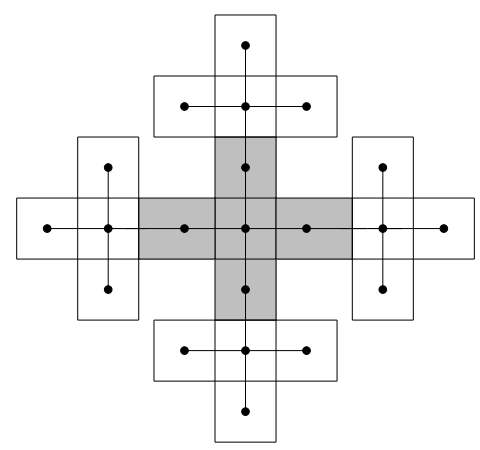

Figure 3: $G_{2}$, four disjoint copies of $G_{1}$ glued with a grey cross $K$, and its weak dual $\mathcal{T}_{G_{2}}$.

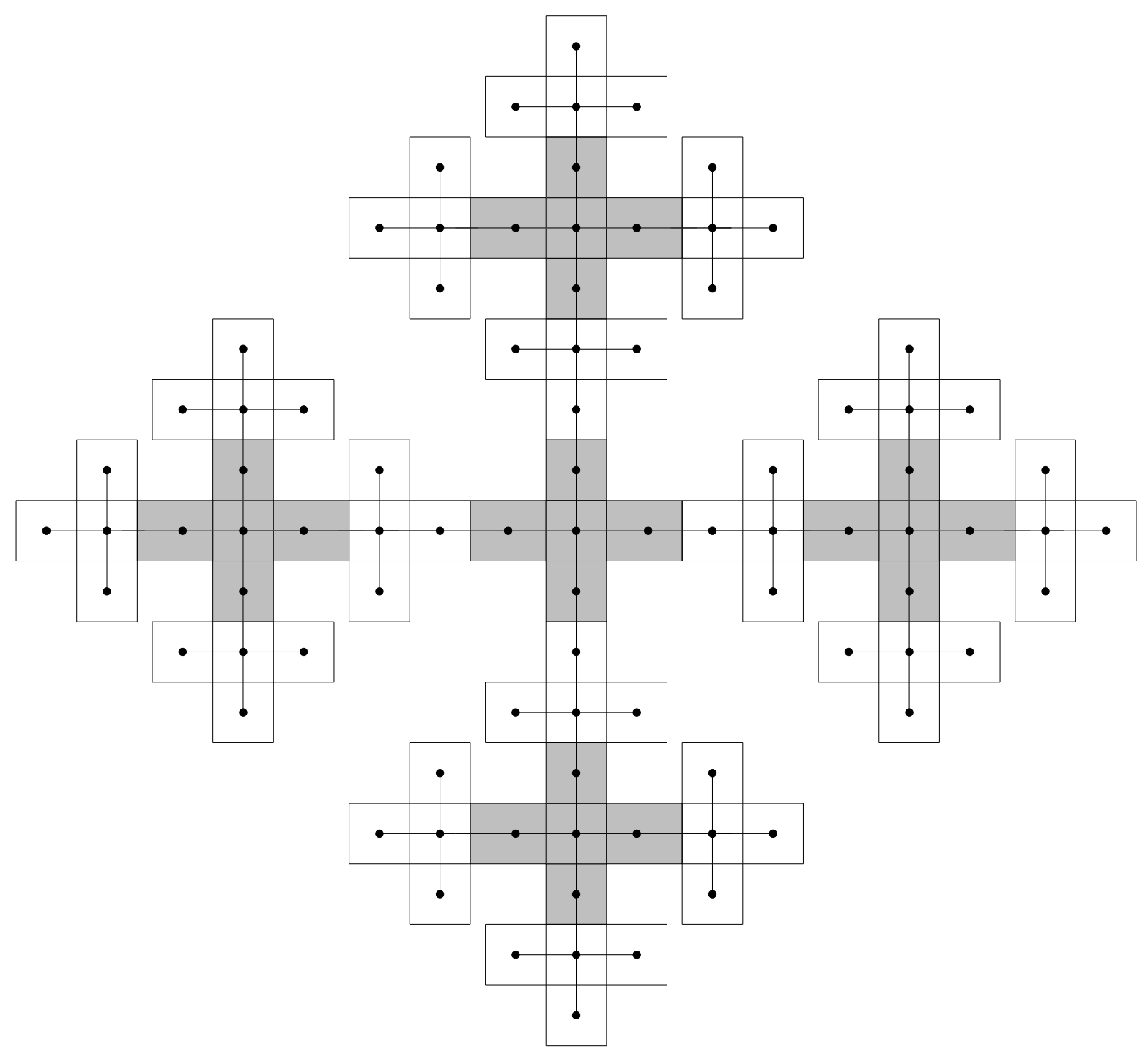

Figure 4: $G_{3}$, four disjoint copies of $G_{2}$ glued with a grey cross $K$, and its weak dual $\mathcal{T}_{G_{3}}$. 
Lemma 1 For each $i \in\{1,2,3,4\}$, let $H_{i}$ be a biconnected outerplanar graph whose weak dual $T_{i}$ has pathwidth $p \geq 1$. The pathwidth of the weak dual of graph $\mathrm{C}\left(\mathrm{H}_{1}, \mathrm{H}_{2}, \mathrm{H}_{3}, H_{4}\right)$ is $p+1$.

We introduce the following definition: for every vertex $v$ of a tree $T$, a branch at $v$ is any maximal subtree which contains a neighbour of $v$ without containing $v$. The following result will be useful to prove Lemma 1.

Theorem 5 (Scheffler [19]) For every integer $p \geq 1$ and every tree $T, \operatorname{pw}(T) \geq p+1$ if and only if there exists a vertex $t$ of $T$ with at least three branches of pathwidth at least $p$.

Proof of Lemma 1. By induction on $p \geq 1$, the result being true for $p=1$. If the pathwidth of each tree $T_{i}$ is $p$, it is not difficult to construct a layout $L$ of the weak dual of $C\left(H_{1}, H_{2}, H_{3}, H_{4}\right)$ with vertex separation $p+1$ : first, label the vertices of $T_{i}$ according to an optimal layout of $T_{1}$. Then, label the vertex $s_{1}$, i.e. define $L\left(s_{1}\right):=\left|V\left(T_{1}\right)\right|+1$. Next, label the vertices of $T_{2}$ (from $L\left(s_{1}\right)+1$ up to $\left.L\left(s_{1}\right)+\left|V\left(T_{2}\right)\right|\right)$ according to an optimal layout of $T_{2}$ such that the unique neighbour of $s_{2}$ in $T_{2}$ is given the biggest integer (such a layout exists by the construction). The next vertex to be labelled is $s_{2}$, and an analoguous labeling is done for the vertices of $T_{3}$ and $s_{3}$. Finally, the vertex $s$ est labelled, then the vertices of $T_{4}$ (according to an optimal layout of $T_{4}$ ) and last the vertex $s_{3}$.

Also, the pathwidth of the weak dual of $C\left(H_{1}, H_{2}, H_{3}, H_{4}\right)$ is more than $p$ by Theorem 5 since the vertex $s$ has four branches with pathwidth $p$.

Lemma 2 For each $i \in\{1,2,3,4\}$, let $H_{i}$ be a biconnected outerplanar graph of pathwidth $p \geq 1$. The vertex separation of the graph $\mathrm{C}\left(\mathrm{H}_{1}, \mathrm{H}_{2}, \mathrm{H}_{3}, \mathrm{H}_{4}\right)$ is at least $\mathrm{p}+2$.

Proof. Consider any layout $L$ of $H:=C\left(H_{1}, H_{2}, H_{3}, H_{4}\right)$. We shall prove that the vertex separation of $(H, L)$ is at least $p+2$. The subgraph of $H$ induced by removing the vertices of the square $S$ is the disjoint union of the four graphs $H_{1}, H_{2}, H_{3}$ and $H_{4}$, each of them having pathwidth $p$. Note that the roles played by those four graphs in this proof are symmetric. Assume that the vertex $a$ such that $L(a)=1$ and the vertex $b$ such that $L(b)=|V(H)|$ are in $V\left(H_{1}\right) \cup V(S)$ and $V\left(H_{1}\right) \cup V\left(H_{2}\right) \cup V(S)$ respectively. By hypothesis, there exists $i \in L\left(V\left(H_{4}\right)\right)$ such that there are $p$ vertices $x$ of $H_{4}$ with $L(x)>$ $i$, each having a neighbour $y$ in $H_{4}$ with $L(y) \leq i$. As a similar integer exists for $H_{3}$, we suppose without loss of generality that there exists a vertex $v \in V\left(H_{3}\right)$ with $L(v)>i$. Let $X:=\cup_{j=1}^{3} V\left(H_{j}\right) \cup V(S)$. Say that a vertex $x \in X$ is an $m$-vertex if $L(x)<i$ and an $M$-vertex if $L(x)>i$. In particular, $a$ is an $m$-vertex and $b$ and $v$ are $M$-vertices. An edge is bad if it links an $m$-vertex to an $M$-vertex. A bad pair is a pair of bad edges that are either disjoint, or incident to the same $m$-vertex. Denote by $Q$ the subgraph of $G$ induced by $X$, and note that the existence of a bad pair in $Q$ implies that vs $(H, L) \geq \operatorname{vs}\left(H_{4}\right)+2=p+2$.

Remark now that $Q$ is 2-connected, so according to the Fan Lemma, there exists in $Q$ two paths $P_{1}$ and $P_{2}$ respectively from $a$ to $b$ and from $a$ to $v$, which are vertex-disjoint except in $a$. Note that $P_{1}$ and $P_{2}$ are both disjoint from $H_{4}$. As $a$ in an $m$-vertex and $b$ and $v$ are $M$-vertices, there exists a bad edge on $P_{1}$ and a bad edge on $P_{2}$, which necessarily from a bad pair (since the only common vertex of $P_{1}$ and $P_{2}$ is $a$, an $m$-vertex). Therefore, the vertex separation of $(H, L)$ is at least $p+2$.

Lemma 3 For each $i \in\{1,2,3,4\}$, let $H_{i}$ be a biconnected outerplanar graph of pathwidth $p \geq 1$. The pathwidth of $\mathrm{C}\left(\mathrm{H}_{1}, \mathrm{H}_{2}, \mathrm{H}_{3}, \mathrm{H}_{4}\right)$ is at most $\mathrm{p}+2$.

Proof. We shall construct a layout of $C\left(H_{1}, H_{2}, H_{3}, H_{4}\right)$ from optimal layouts $L_{i}$ of $H_{i}, i \in\{1,2,3,4\}$. Start by labelling all the vertices of $H_{4}$ according to $L_{4}$. The vertex separation never exceeds $p+2$, 
since the only unlabelled vertices not in $H_{4}$ that might have labelled neighbours are $\beta$ and $\gamma$. By the construction, the optimal layout $L_{1}$ of $H_{1}$ can be chosen such that $L_{1}\left(x_{1}\right)<L_{1}\left(y_{1}\right)$. Label the vertices of $H_{1}$ until the vertex $x_{1}$ is labelled. As previously, the vertex separation does not exceed $p+2$ when doing so. Now, label the vertex $\beta$, which does not change the vertex separation, as $\beta$ has exactly one unlabelled vertex, $\alpha$. Now go on labelling the vertices of $H_{1}$ according to $L_{1}$. The vertex-separation still does not exceed $p+2$, the only unlabelled vertices not in $H_{1}$ with labelled neighbours being $\alpha$ and $\gamma$. By the construction again, the layout $L_{2}$ of $H_{2}$ can be chosen such that $L_{2}\left(x_{2}\right)<L_{2}\left(y_{2}\right)$. Therefore we can apply the same procedure to label the vertices of $H_{2}$ : first label them until $x_{2}$ is labelled, then label the vertex $\alpha$ and finish labelling the vertices of $\mathrm{H}_{2}$. At last, label the vertices of $\mathrm{H}_{3}$ (the vertex separation does not exceed $p+2$ when doing so, since the only unlabelled vertices with labelled neighbours not in $H_{3}$ are $\delta$ and $\gamma$ ), and then label the vertices $\delta$ and $\gamma$. The obtained layout has vertex separation at most $p+2$.

Proof of Theorem 3. The proof is by induction on $p \geq 1$. If $p=1$, two adjacent squares and $G_{1}$ give the desired result when $k=1$ and $k=2$ respectively.

Suppose that the result is true for $p-1 \geq 1$, and let $k \in\{1,2, \ldots, p+1\}$. First, let $k=1$ : as is well known, there exist biconnected outerplanar graphs of pathwidth $p+1$ whose weak dual has pathwidth $p$. If $k \in\{2,3, \ldots, p+1\}$, then $k-1 \in\{1,2, \ldots, p\}$ so, by the induction hypothesis, there exists a biconnected outerplanar $H$ of pathwidth $(p-1)+(k-1)$ whose weak dual has pathwidth $p-1$. Then by Lemmata 1, 2 and 3, $C(H, H, H, H)$ has pathwidth $p+k$ and its weak dual has pathwidth $p$, as desired.

Now we show how the family $\left(G_{p}\right)_{p \geq 1}$ can be used to also disprove Conjecture 3 .

First, observe that the pathwidth of a multigraph $G$ is equal to the pathwidth of its underlying simple graph, denoted $\mathcal{U}(G)$.

Let $u v$ be an edge of a 2-connected planar graph $G$. Denote $F_{1}$ and $F_{2}$ the two faces incident to $u v$, and $f_{1}$ and $f_{2}$ the corresponding vertices of $G^{*}$. To subdivide $i$ times the edge $u v$ (i.e. to replace it by an induced path of length $i+1$ ) leads to replace the edge $f_{1} f_{2}$ in $G^{*}$ by $i+1$ parallel edges.

Now, consider $G_{p}$ and an embedding of $G_{p}^{*}$ such that $G_{p}^{* *} \simeq G_{p}$. Call $o$ the vertex corresponding to the external face of $G_{p}$ in $G_{p}^{*}$. Let $H_{p}$ be the grah obtained by subdividing each edge of $G_{p}^{*}$ incident to $o$ (i.e. by replacing it by an induced path of length two). Notice that $H_{p}$ is a simple 2-connected planar graph. According to the preceding remarks, $\mathcal{U}\left(H_{p}^{*}\right) \simeq G_{p}$, and hence $\operatorname{pw}\left(H_{p}^{*}\right)=\operatorname{pw}\left(G_{p}\right)$.

For every face $F$ of $G_{p}$, let $m(F)$ be the number of edges of $F$ incident to the unbounded face. Let $m:=\max _{F}(m(F))$.

Lemma $4 \mathrm{pw}\left(H_{p}\right) \leq \operatorname{pw}\left(G_{p}^{*}\right)+m=\operatorname{pw}\left(G_{p}^{*}\right)+3$

Proof. It is clear by the definition of $G_{p}$ that $m=3$. Let $l$ be an optimal layout of $G_{p}^{*}$ (i.e. a layout of minimum vertex-separation), and let construct a layout of $H_{p}$ of width at most pw $\left(G^{*}\right)+m$. Let us label every vertex $v$ belonging to both $H_{p}$ and $G_{p}^{*}$ with $(l(v), 0)$. Now, if the vertex labelled $(l(v), 0)$ has $j$ unlabelled neighbours, label them $(l(v), 1), \cdots,(l(v), j)$. The obtained labeling surely has vertex separation at most $\mathrm{pw}\left(G_{p}^{*}\right)+m$.

If Conjecture 2 is true, then $\operatorname{pw}\left(H_{p}\right) \geq \operatorname{pw}\left(H_{p}^{*}\right)-1=\operatorname{pw}\left(G_{p}\right)-1$. However, by lemma 4, $\operatorname{pw}\left(H_{p}\right) \leq \mathrm{pw}\left(G_{p}^{*}\right)+3$ and so $\mathrm{pw}\left(G_{p}^{*}\right)+3 \geq \mathrm{pw}\left(G_{p}\right)-1$ which is false for $p>6$. 


\section{Upper bound}

We shall present in this section an algorithm which, given a biconnected outerplanar graph $G$, computes a layout of $G$ with vertex separation at most $2 \mathrm{pw}\left(\mathcal{T}_{G}\right)+1$. As $\operatorname{pw}\left(\mathcal{T}_{G}\right)=\operatorname{pw}\left(G^{*}\right)-1$ for any biconnected outerplanar $G$ (see [3]), this establishes Theorem 4.

First, recall that a caterpillar is a tree in which a single path, the spine, is incident to (or contains) every edge. The caterpillars are the only trees of pathwidth one: every caterpillar has surely pathwidth one, and if a tree $T$ is not a caterpillar, then it contains a spider with three legs of length two (see Figure 5). But such a tree has pathwidth at least two by Theorem 5.

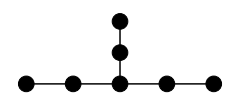

Figure 5: A spider with three legs of length two.

Proposition 1 Let $G$ be a biconnected outerplanar graph whose weak dual is a caterpillar. Then $G$ has pathwidth at most three.

Proof. Here is a layout of the vertices of $G$ with vertex separation at most three. Let $P:=v_{1} v_{2} \ldots v_{k}$ be a longest path of $\mathcal{T}_{G}$. Denote by $F_{i}$ the face of $G$ corresponding to the vertex $v_{i}, i \in\{1,2, \ldots, k\}$. Label by 1 a vertex $v$ of $F_{1}$ of degree two (such a vertex exists as $G$ is outerplanar and $v_{1}$ is a leaf of $T)$. Then recursively label every vertex of $F_{1}$ of degree two which is adjacent to a labelled vertex.

Now, apply the following procedure in which we suppose that $V\left(F_{i-1}\right) \cap V\left(F_{i}\right)=\left\{x_{i}, y_{i}\right\}$ and $V\left(F_{i}\right) \cap V\left(F_{i+1}\right)=\left\{x_{i+1}, y_{i+1}\right\}$, see Figure 6 .

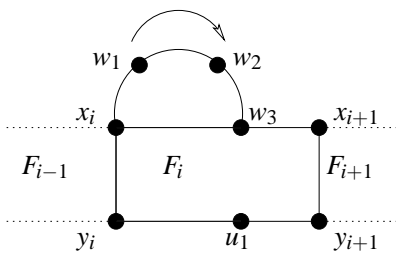

Figure 6: Vertices for step $i$.

1: for $i=2$ to $k-1$ do

2: let $P:=x_{i} w_{1} \ldots w_{j} x_{i+1}$ be the path of $G$ from $x_{i}$ to $x_{i+1}$ consisting of edges incident to the unbounded face. Label the vertices of $P$ from $x_{i}$ to $w_{k}$

3: let $P^{\prime}:=y_{i} u_{1} \ldots u_{t} y_{i+1}$ be the path of $G$ from $y_{i}$ to $y_{i+1}$ consisting of edges incident to the unbounded face. Label the vertices of $P^{\prime}$ from $y_{i}$ to $u_{t}$

4: end for

Last, label the vertices clockwise from $x_{k}$ to $y_{k}$.

The obtained layout surely has vertex separation at most three.

The procedure given in the preceding proof actually achieves an optimal layout of the corresponding graph. Indeed, such a graph has pathwidth two if its weak dual is path, and pathwidth three otherwise. Note also that the time complexity of the procedure is linear.

We will use the following result about the pathwidth of trees. 
Theorem 6 (Ellis, Sudborough and Turner [8]) For any tree $T$, and any integer $p \geq 2$, $p w(T) \leq p$ if and only if there is a path $P$ such that every connected component of the forest induced by the vertices of $V(T) \backslash V(P)$ has pathwidth at most $p-1$.

We consider the recursive procedure given by Algorithm 1. It computes a layout of $G$ stored in the list $l$, which is initialised by $l(v):=\infty$ for every vertex $v \in V(G)$ (this means that all vertices are unlabelled at the beginning).

Notice that what is done in lines 16-17 and 32-33 is equivalent to label all the vertices of $H$ except $y$ (or $y^{\prime}$ respectively), and to keep $s$ updated.

The following lemma suffices to establish Theorem 4.

Lemma 5 For any biconnected outerplanar graph $G$ whose weak dual $T$ has pathwidth $p$, the procedure Layout of Algorithm 1 returns a layout with vertex separation at most $2 p+1$.

Proof. Algorithm 1 clearly assigns a unique label to every vertex of $G$.

For the vertex separation of the obtained layout, the proof is by induction on the pathwidth $p$ of $T$. If $p$ is one, then $T$ is a caterpillar and Proposition 1 gives the conclusion.

Suppose now that for every biconnected outerplanar graph whose weak dual has pathwidth at most $p-1 \geq 1$, the procedure Layout of Algorithm 1 returns a layout with vertex separation at most $2 p-1$. Let us prove that the obtained layout for $G$ has pathwidth at most $2 p+1$.

Stop the labelling of $G$ at any moment and denote by $F$ the set of unlabelled vertices with a labelled neighbour. If no subgraph $H$ has been labelled yet, then the set $F$ consists of $x$ and $x^{\prime}$, so its size is at most $2 p+1$. If a subgraph $H$ has just been labelled, then $F$ consists of two vertices, namely $x^{\prime}$ and $y$ or $x$ and $y^{\prime}$.

Suppose now that a subgraph $H$ is being labelled. Without loss of generality, say that its intersection with the current face $F_{i}$ is $\{x, y\}$. There is only one vertex of $F$ not in $H$, namely $x^{\prime}$. Therefore, if $|F \cap V(H)| \leq 2 p$ we have $|F| \leq 2 p+1$ as wanted. As the vertex separation of the layout used to label $H$ is at most $2 p-1$, the only problem that might occur is if $|F \cap(V(H) \backslash\{x, y\})|=2 p-1$, and $x, x^{\prime}$ and $y$ also belong to $F$. This implies that $y$ was requested to be labelled in the original layout $l$ used for $H$, but kept unlabelled as indicated in the algorithm. But in this case, in the labelling $l$ of $H$, the vertex $x$ is unlabelled, and has at least a labelled neighbour, $y$. So the number of unlabelled vertices of $H$ with a labelled neighbour in $H$ is $|F \cap(V(H) \backslash\{x, y\})|+1=2 p$, a contradiction.

As one can see in the preceding proof, the subgraphs $H$, labelled in lines 16 and 32, can actually be labelled by any layout with vertex separation at most $2 p-1$.

Corollary 2 For any biconnected outerplanar graph $G$, $\mathrm{pw}\left(\mathcal{T}_{G}\right)+1 \leq \mathrm{pw}(G) \leq 2 \mathrm{pw}\left(\mathcal{T}_{G}\right)+1$. Furthermore the bounds are tight.

As proved in [19], the pathwidth of a tree with $f$ vertices is less than $\log _{3}(2 f+1)$. Thus we have the following corollary.

Corollary 3 The pathwidth of any biconnected outerplanar graph $G$ with $f$ inner faces is less than $2 \log _{3}(2 f+1)+1$.

Proposition 2 The time complexity of Algorithm 1 is $O(n \log (n))$. 


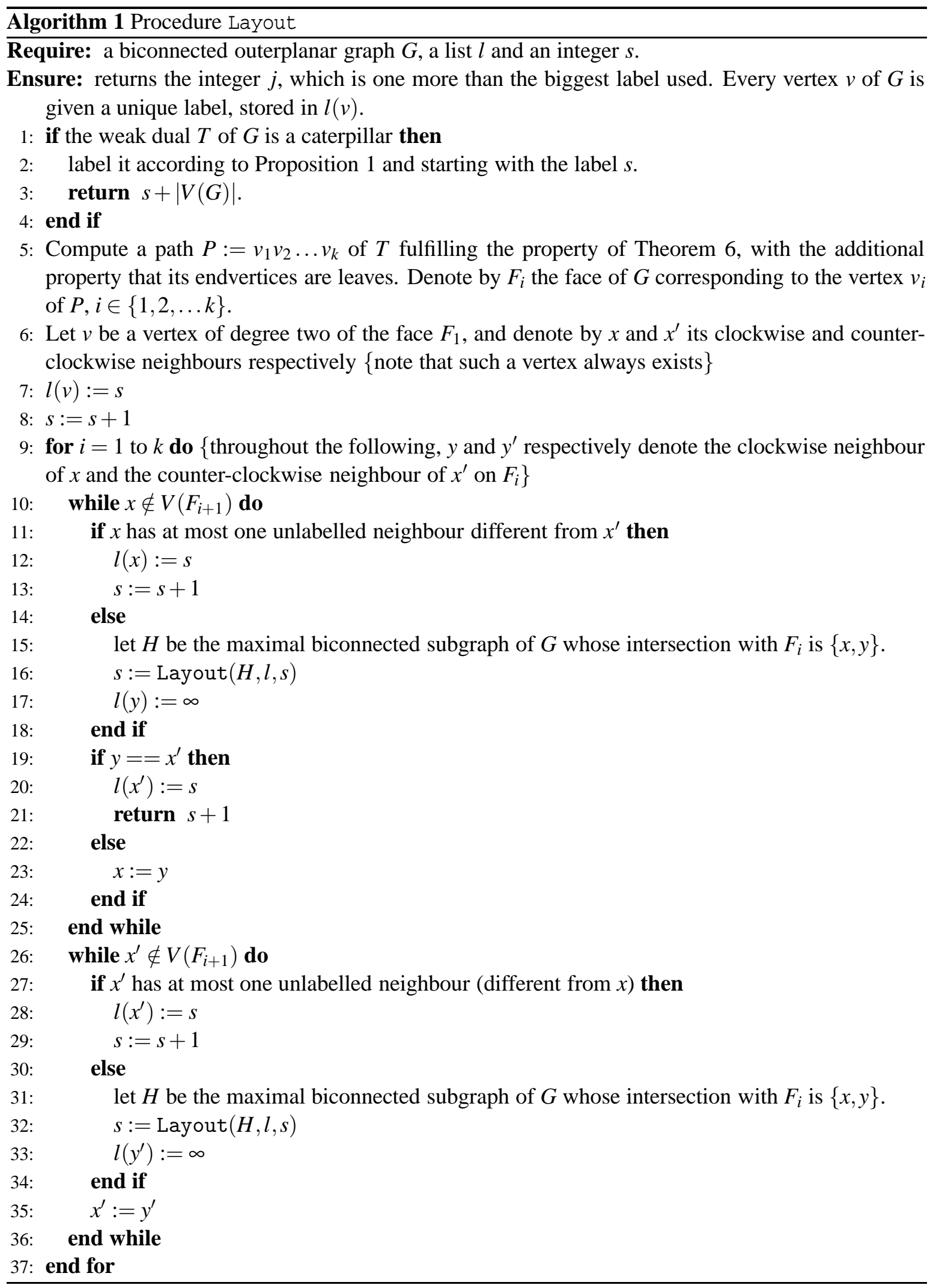


Proof. It is easy to see that the time complexity of Algorithm 1 depends mainly on the recursive calls and on the time complexity of line 5 (since computing the weak dual of a biconnected outerplanar graph and determining whether a tree is a caterpillar is linear in time, as is the procedure of Proposition 1). We first show that the time complexity of Algorithm 1 without line 5 is linear.

For that, remark that a node $x$ of face $F_{i}$ is labelled directly during the processing of face $F_{i}$ if it has at most one unlabelled neighbour different from $x^{\prime}$, otherwise during the recursive call, or it will be considered again during the processing of face $F_{i+1}$. So a node $x$ is considered once in each inner face to which it belongs, that is its degree minus one. So altogether we have $2(|E|-|V|)$ steps, which is equal to $2(f-1)$ using Euler's formula for planar graphs, where $f$ is the total number of inner faces. Since the number of faces of a biconnected outerplanar graph is smaller that its number of vertices, the time complexity of Algorithm 1 without line 5 is linear.

The computation of a path $P$ fulfilling the property of Theorem 6, with the additional property that its endvertices are leaves, is similar in style to the techniques used in $[8,22,14]$ on trees to compute vertex separation, cutwidth and search number. Thus it can be done in linear-time. Furthermore, the pathwidth of a tree with $f$ vertices being less than $\log _{3}(2 f+1)$ [19], the computation of all paths takes time $O\left(f \log _{3}(2 f+1)\right)$, that is $O(n \log (n))$.

Theorem 4 clearly provides a linear-time algorithm to approximate the pathwidth of a biconnected outerplanar graph $G$ since computing the dual tree of $G$ and its pathwidth can both be done in lineartime. A corresponding layout is given by Algorithm 1, whose time complexity is $O(n \log (n))$. As noted in [3], there exist trees and outerplanar graphs for which a straight representation of a layout needs $\Omega(n \log (n))$ in time just to be written. Skodinis [20] developed a representation so that path decompositions (and layouts) can be written in linear-time. We did not try to use it for Algorithm 1 but we suspect that it can be used to precompute all paths in linear-time and thus reduce the complexity to $O(n)$. For unicyclic graphs, which in particular are outerplanar, Ellis and Markov [7] gave an algorithm that computes the vertex separation along with a corresponding linear layout in time $O(n \log (n))$.

Corollary 4 For any biconnected outerplanar graph $G$, Algorithm 1 provides in time $O(n \log (n))$ a layout of $G$ with vertex separation at most $2 \mathrm{pw}(G)-1$.

\section{Conclusion}

We strengthened the previously known relation between the pathwidth of a biconnected outerplanar graph and the pathwidth of its dual. We did so in an algorithmic way and thus obtained a new approximation algorithm. We established the tightness of our bound, thereby disproving two of conjectures of Bodlaender and Fomin $[3,10]$, and moreover we showed that all cases in the interval happen.

To conclude, we note here that, according to [10], Conjecture 3 is implied by another conjecture of Fomin [10]. We need two new definitions to state it.

Given an edge-ordering $\sigma$ of $G=(V, E)$, let $\delta(i)$ be the number of vertices incident to at least two edges $e, e^{\prime}$ such that $\sigma(e) \leq i$ and $\sigma\left(e^{\prime}\right)>i$. The linear width of $(G, \sigma)$ is the maximum of $\delta(i), i \in\{1,2, \ldots,|E|\}$. The linear width of $G$, denoted by $\operatorname{lw}(G)$, is the minimum of the linear width of $(G, \sigma)$ taken over all the edge-orderings $\sigma$. Notice that if $G$ has minimum degree at least two, then $\operatorname{pw}(G) \leq \operatorname{lw}(G) \leq \operatorname{pw}(G)+1$. For a planar graph $G$, a split $H$ of $G$ is a graph obtained by a sequence of the following operations: take a vertex $v$, partition its neighbourhood in two sets $M$ and $N$, replace $v$ by two new vertices $x, y$. Link $x$ to $M \cup\{y\}$ and $y$ to $N$. 
Conjecture 4 (Fomin [10]) For every planar graph $G$, there exists a planar split $H$ of maximum degree three such that $\operatorname{lw}(H)=\operatorname{lw}(G)$.

As Conjecture 3 is disproved in Section 2, Conjecture 4 does not hold.

We end with a question. Fomin and Thilikos [9] proved that, for every 3-connected planar graph $G$, the pathwidth of $G^{*}$ is at most 6 times the pathwidth of $G$. Amini, Huc and Pérennes [1] showed that this bound can be reduced to three (and even to two if $G$ is 4-connected).

Problem 1 Is there a constant $c$ such that, for every 2-connected planar graph $G$,

$$
\frac{1}{2} \mathrm{pw}\left(G^{*}\right)-c \leq \mathrm{pw}(G) \leq 2 \mathrm{pw}\left(G^{*}\right)+c ?
$$

If the answer is positive, the multiplicative factor two would be optimal by Corollary 1.

\section{References}

[1] O. Amini, F. Huc, and S. Pérennes. On the pathwidth of planar graphs. Research report, INRIA Research Report HAL-00082035, July 2006.

[2] H. L. Bodlaender. A partial $k$-arboretum of graphs with bounded treewidth. Theor. Comput. Sci., 209(1-2):1-45, 1998.

[3] H. L. Bodlaender and F. V. Fomin. Approximation of pathwidth of outerplanar graphs. $J$. Algorithms, 43(2):190-200, 2002.

[4] H. L. Bodlaender and T. Kloks. Efficient and constructive algorithms for the pathwidth and treewidth of graphs. J. Algorithms, 21(2):358-402, 1996.

[5] V. Bouchitté, F. Mazoit, and I. Todinca. Chordal embeddings of planar graphs. Discrete Math., 273(1-3):85-102, 2003. EuroComb’01 (Barcelona).

[6] J. Díaz, J. Petit, and M. Serna. A survey of graph layout problems. ACM Computing Surveys, 34(3):313-356, 2002.

[7] J. Ellis and M. Markov. Computing the vertex separation of unicyclic graphs. Inform. and Comput., 192(2):123-161, 2004.

[8] J. A. Ellis, I. H. Sudborough, and J. S. Turner. The vertex separation and search number of a graph. Inform. and Comput., 113(1):50-79, 1994.

[9] F. Fomin and D. M. Thilikos. On self duality of pathwidth in polyhedral graph embeddings. Report in Informatics 316, University of Bergen, March 2006.

[10] F. V. Fomin. Pathwidth of planar and line graphs. Graphs and Combinatorics, 19(1):91-99, 2003.

[11] R. Govindan, M. A. Langston, and X. Yan. Approximating the pathwidth of outerplanar graphs. Inform. Process. Lett., 68(1):17-23, 1998.

[12] N. G. Kinnersley. The vertex separation number of a graph equals its pathwidth. Inform. Process. Lett., 42(6):345-350, 1992. 
[13] D. Lapoire. Structuration des graphes planaires. PhD thesis, Université de Bordeaux, France, 1999.

[14] N. Megiddo, S. L. Hakimi, M. R. Garey, D. S. Johnson, and C. H. Papadimitriou. The complexity of searching a graph. J. Assoc. Comput. Mach., 35(1):18-44, 1988.

[15] S. L. Mitchell. Linear algorithms to recognize outerplanar and maximal outerplanar graphs. Inform. Process. Lett., 9(5):229-232, 1979.

[16] B. Reed. Treewidth and tangles: an new connectivity measure and some applications. In R. A. Bayley, editor, Surveys in Combinatorics, pages 87-162. Cambridge University Press, 1997.

[17] N. Robertson and P. D. Seymour. Graph minors. I. Excluding a forest. J. Combin. Theory Ser. $B, 35(1): 39-61,1983$.

[18] N. Robertson and P. D. Seymour. Graph minors. III. Planar tree-width. J. Combin. Theory Ser. $B, 36(1): 49-64,1984$.

[19] P. Scheffler. A linear algorithm for the pathwidth of trees. In R. Henn R. Bodendiek, editor, Topics in Combinatorics and Graph Theory, pages 613-620. Physica-Verlag Heidelberg, 1990.

[20] K. Skodinis. Construction of linear tree-layouts which are optimal with respect to vertex separation in linear time. J. Algorithms, 47(1):40-59, 2003.

[21] M. M. Sysło. Characterisations of outerplanar graphs. Discrete Math., 26(1):47-53, 1979.

[22] M. Yannakakis. A polynomial algorithm for the min-cut linear arrangement of trees. J. Assoc. Comput. Mach., 32(4):950-988, 1985. 